\title{
CONTENTS
}

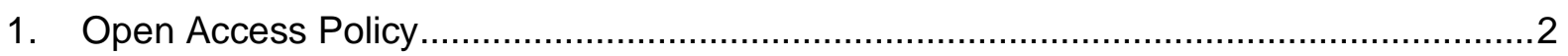

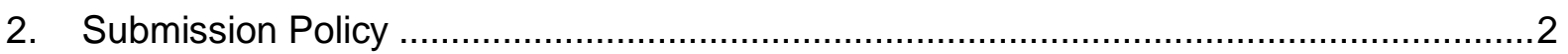

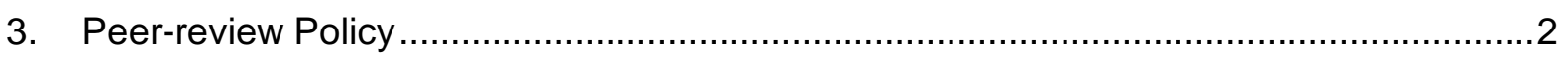

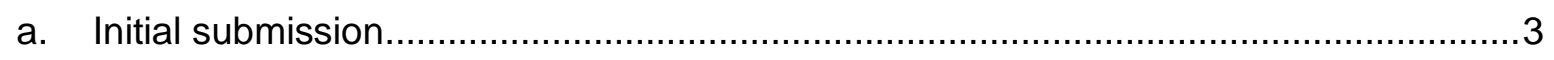

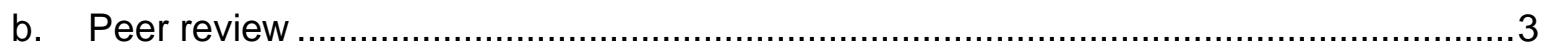

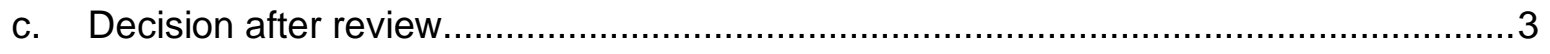

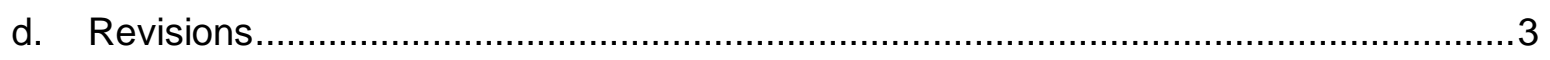

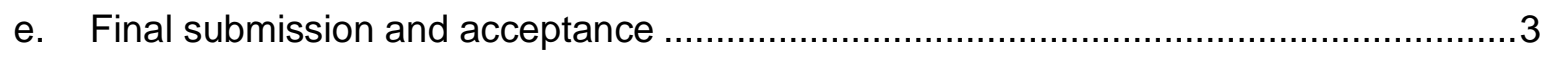

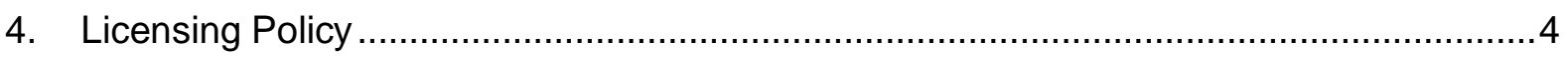

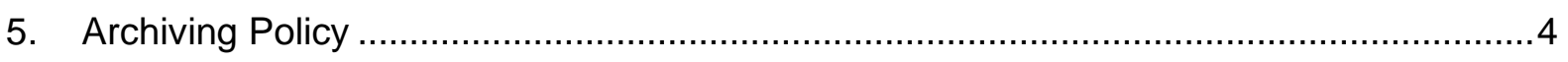

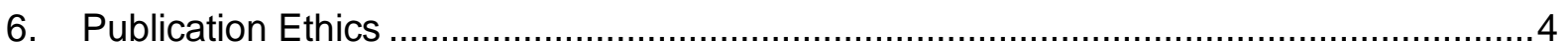

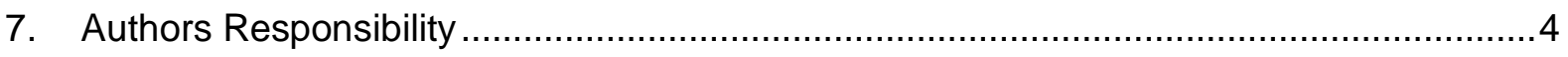

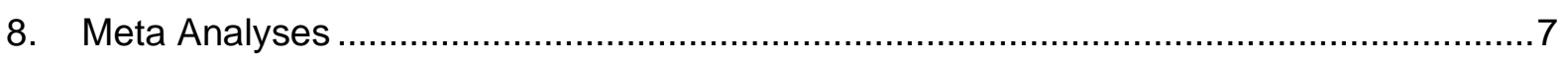

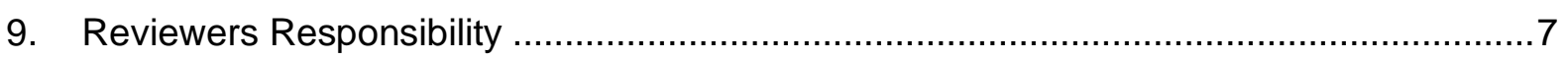

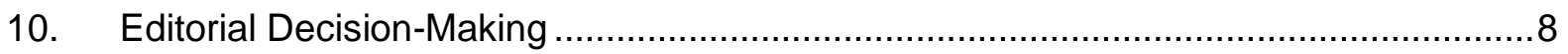

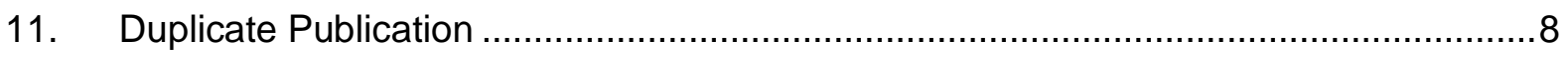

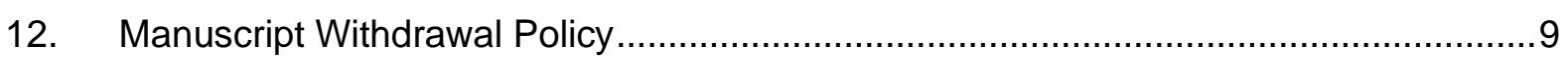

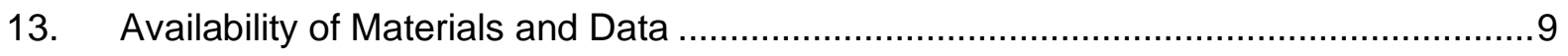

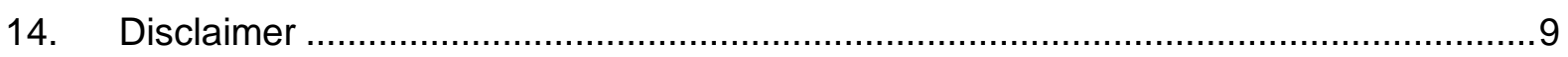

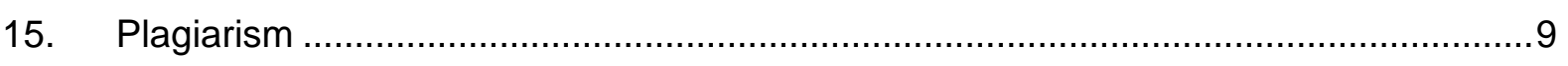

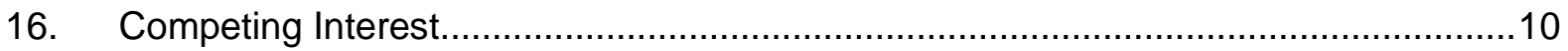

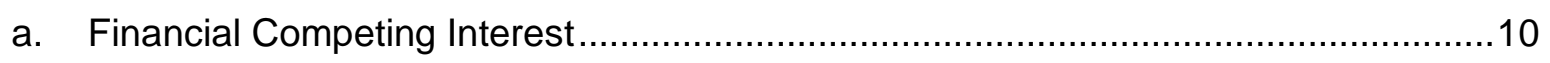

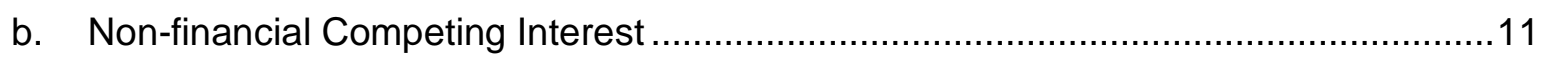

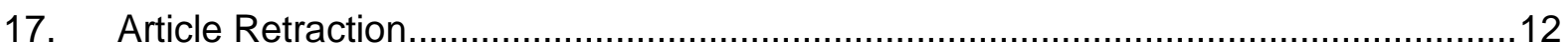


Geo Eye (GE) had adopted the following journal policies.

\section{Open Access Policy}

In order to ensure maximum reach of Geo Eye contents, we have adopted the open access policy, meaning that, all the articles published in Geo Eye are made freely accessible online immediately after publication in an easily readable format, without any subscription or registration barrier.

\section{Submission Policy}

- We welcome original research articles, review articles, educational articles, case reports and short communications.

- When you submit a manuscript to GE, we will take it to imply that the manuscript has not already been published or submitted elsewhere. You may not submit your manuscript elsewhere while it is under consideration with GE.

- GE reserve the right to accept or reject an article. A paper can be rejected even after it has been accepted if it becomes apparent that there are serious problems with its scientific content, or our publishing policies have been violated.

- A copy of approval by human ethics committee or animal ethics committee (in case of animal studies) is mandatory for original research articles.

\section{Peer-review Policy}

All the manuscripts submitted to Geo Eye will be subjected to double blinded peer review process;

- The manuscript will be reviewed by two suitable experts in respective subject area.

- The reports of both the reviewers will be considered while deciding on acceptance/revision or rejection of a manuscript.

- Editor-In-Chief will make the final decision, based on reviewer's comments.

- Editor-In-Chief can ask one or more of the advisory board members for their suggestions upon a manuscript, before making the final decision.

- Associate and assistant editors provide the administrative support to maintain the integrity of peer review process.

- In case, authors challenge editor's negative decision with suitable arguments, the manuscript can be sent to one more reviewer and the final decision will be made based upon his recommendations.

- Under any circumstances, the correspondence by the editorial board members or reviewers except Editor-in-chief and Editor with the authors is entertained. If there is evidence of such happening, then the paper will be summarily rejected. 
a. Initial submission

Once submitted, your manuscript will be assigned to a member of our Editorial Board, who will read the paper and decide whether it is appropriate for the journal. Manuscripts that are within scope and seem, on initial assessment, to be technically sound and scientifically valid, will be sent to external reviewers. Copies of any papers containing similar or related work under consideration or in press at other journals must be included with the submission.

\section{b. Peer review}

At the submission stage, authors may indicate a limited number of scientists who should not review the paper. Excluded scientists must be identified by name. Authors may also suggest potential reviewers; these suggestions are often helpful, but they are not always followed. By policy, referees are not identified to the authors, except at the request of the referee.

\section{c. Decision after review}

After considering the reviewer reports the Editorial Board Member will make one of the following decisions:

- Accept outright

- Request a minor revision, where authors revise their manuscript to address specific concerns

- Request a major revision, where authors revise their manuscript to address significant concerns and perhaps undertake additional work

- Reject outright

\section{d. Revisions}

In cases where the referees or Editorial Board Member has requested changes to the manuscript, you will be invited to prepare a revision. The decision letter will specify a deadline for submission of a revised manuscript. Once resubmitted, the manuscript may then be sent back to the original referees or to new referees, at the Editorial Board Member's discretion.

A revised manuscript should be submitted not as a new manuscript. The revision should also be accompanied by a point-by-point response to referees explaining how the manuscript has been changed.

\section{e. Final submission and acceptance}

When all editorial issues are resolved, your paper will be formally accepted for publication. After acceptance, authors are sent proofs of their manuscript but only changes to the title, author list or scientific errors will be permitted. All 
corrections must be approved by the publishing team.

Manuscripts should be formatted according to the instructions given in author guidelines.

\section{Licensing Policy}

To fully realize the potential of open access to research literature, barriers to reuse need to be removed. The Creative Commons (CC) licenses have emerged as an effective legal instrument to achieve this.

Instead of transferring rights exclusively to publishers (the approach usually followed in subscription publishing), authors grant a non-exclusive license to the publisher to distribute the work, and all users and readers are granted rights to reuse the work.

CC-BY allows for unrestricted reuse of content, subject only to the requirement that the source work is appropriately attributed. The CC-BY license thereby requires that authors are given appropriate credit for their work, as explained in a recent post from Creative Commons

(http://wiki.creativecommons.org/BIS committee UK OA Policy).

\section{Archiving Policy}

This journal utilizes the AWS system to create permanent archives of the journal for purposes of preservation and restoration.

\section{Publication Ethics}

The GA is an electronic Double Blind peer reviewed journal upholding the highest standards. Articles not in accordance with publication ethics and malpractices will be removed from publication if detected at any time. Plagiarism and research fabrication such as making up of data, manipulation of existing data, tables etc. and ethical clearance on the use of humans or animals for the study will also be checked. The journal reserves the right to use plagiarism detecting software to screen submitted papers at any time and suspected plagiarism or duplicate publishing will be reported immediately.

\section{Authors Responsibility}

The primary affiliation for each author should be the institution where the majority of their work was done. If an author has subsequently moved, the current address may 
also be stated. GE hold no responsibility with regard to jurisdictional claims in published institutional affiliations.

Authorship provides credit for a researcher's contributions to a study and carries accountability. Each author is expected to have made substantial contributions to the conception or design of the work or the acquisition, analysis, or interpretation of data.

All authors to have agreed both to be personally accountable for the author's own contributions and to ensure that questions related to the accuracy or integrity of any part of the work, even ones in which the author was not personally involved, are appropriately investigated, resolved, and the resolution documented in the literature.

The corresponding (submitting) author is solely responsible for communicating with GE and for managing communication between co-authors. Before submission, the corresponding author ensures that all authors are included in the author list, its order has been agreed by all authors, and that all authors are aware that the paper was submitted.

After acceptance, the proof is sent to the corresponding author. He/She is responsible for the accuracy of all content in the proof, in particular that names of co-authors are present and correctly spelt, and that addresses and affiliations are current.

GE regards the corresponding author as the point of contact for queries about the published paper. It is this author's responsibility to inform all co-authors of matters arising and to ensure such matters are dealt with promptly. This author does not have to be the senior author of the paper or the author who actually supplies materials; this author's role is to ensure enquiries are answered promptly on behalf of all the coauthors.

GE treats the submitted manuscript and all communication with authors and referees as confidential. Authors must also treat communication with GE as confidential. Correspondence, referee reports and other confidential material must not be posted on any website or otherwise publicized without prior permission from the GE.

Authors may suggest potential reviewers but please keep in mind that we are not obliged to follow these recommendations. The decision of the Editorial Board Members on the choice of referees is final.

Material submitted to GE should not be discussed with the media, except in the case permitted by the editorial board in writing. 
Authors must ensure that

- Their work is original

- Must not have been published elsewhere

- Other authors, contributors or sources should be appropriately credited

- They are responsible for language editing before submission

- Their work has not been copied or plagiarized in whole or part from any other work

- The financial support and conflict of interest for the project / research work if any should be disclosed

- They are obliged to notify the editor if any error or inaccuracy is discovered and co- operate with the editor to correct the same

Articles should be submitted using online procedures. Simultaneous publication in more than one publication is a breach of publication ethics.

Committee on Publication Ethics (COPE) during the 2nd World Conference on Research Integrity in Singapore in 2010 has developed the below mentioned guidelines. Sciresol Journals, as a publisher follows COPE, WAME and has adopted COPE's best practice guidelines for dealing with ethical issues in a journal. The Journal Members (Advisory Board, editors and the journal manager) have consented to adopt the COPE as it meets the purpose and objective of the Journal.

The following is a summary of COPE's international standards for authors of scholarly research publications and describes responsible research reporting practice. The comprehensive guidelines can be downloaded here

- The research being reported should have been conducted in an ethical and responsible manner and should comply with all relevant legislation.

- Researchers should present their results clearly, honestly, and without fabrication, falsification or inappropriate data manipulation.

- Researchers should strive to describe their methods clearly and unambiguously so that their findings can be confirmed by others.

- Researchers should adhere to publication requirements that submitted work is original, is not plagiarized, and has not been published elsewhere.

- Authors should take collective responsibility for submitted and published work.

- The authorship of research publications should accurately reflect individuals' contributions to the work and its reporting.

- Funding sources and relevant conflicts of interest should be disclosed.

They must ensure that

- The submitted manuscript undergoes Double - blind peer review before publishing

- They will strive to prevent any conflict of interest between the author and editorial and review personnel 
- All the information related to the submitted manuscript is kept confidential prior to publishing

\section{Meta Analyses}

Articles reporting meta-analyses must be accompanied by a completed PRISMA (Preferred Reporting Items for Systematic Reviews and Meta-Analyses) checklist and flow diagram, available at www.prisma-statement.org.

\section{Reviewers Responsibility}

They must ensure that

- Reviews of the manuscript is completed and provides suitable comments for improvement.

- Promptness in notifying the editor about his inability to review the manuscript.

- Maintains confidentiality about the manuscript obtained for review, by not discussing with others.

- Standards of Objectivity preserved by reviewing the manuscripts objectively with clear views and supporting arguments and without any personal criticism.

- Acknowledgement of Sources.

- All References not cited by author should be removed. All citations should be accompanied with any previously reported observation, derivation or argument.

- The editors should be intimated for any overlap of the reviewed manuscript with any other published paper.

- Disclosures and Conflict of Interest- manuscripts having conflict of interest resulting from competitive, collaborative or any other relationships or connections with institutions connected to the paper will not be considered.

In case, plagiarism is detected during review/editorial process, such manuscript(s) will be rejected immediately and we can also consider other steps depending upon the seriousness of the case. If the plagiarism is proven after publication, such manuscript(s) will be removed from our website and appropriate announcement will be placed in this regards.

Further, Geo Eye can bring such instances in notice of author's funding agencies, author's institutes (where they work) and to the original authors whose work has been plagiarized. Please report the plagiarism to info@sciresol.com 


\section{Editorial Decision-Making}

Decisions about types of correction are made by the journal's in-house editors, sometimes with the advice of referees, Editorial Advisory Panel or Editorial Board Members. This process involves consultation with the authors of the paper, but the in-house editors make the final decision about whether an amendment is required and the category in which the amendment is published.

Authors sometimes request a correction to their published contribution that does not affect the contribution in a significant way or impair the reader's understanding of the contribution In these cases, the fact that a correction has been made is stated in a footnote so that readers are aware that the originally published text has been amended.

\section{Duplicate Publication}

Material submitted to GE must be original and not published or submitted for.

Duplicate publication occurs when an author re-uses substantial parts of his or her own published work without providing the appropriate references. This can range from getting an identical paper published in multiple journals, to slicing of the paper, where authors add small amounts of new data to a previous paper.

GE is happy to consider submissions containing material that has previously formed part of a Ph.D. or other academic thesis which has been published according to the requirements of the institution awarding the qualification.

GE allows publication of meeting abstracts before the full contribution is submitted. Such abstracts should be included with the submission and referred to in the cover letter accompanying the manuscript. This policy does not extend to meeting abstracts and reports available to the media or which are otherwise publicized outside the scientific community during the submission and consideration process.

Publishing work in conference proceedings is common in some research communities. We are happy to consider submissions containing material that has been published in a conference proceedings paper. However, the submission should provide a substantial extension of results, analysis, conclusions and/or implications over the conference proceedings paper. Authors must provide details of the conference proceedings paper with their submission including a relevant citation in the submitted manuscript. Authors must obtain all necessary permissions to re-use previously published material and attribute appropriately. 
If an author of a submission is re-using a figure or figures published elsewhere, or that is copyrighted, the author must provide documentation that the previous publisher or copyright holder has given permission for the figure to be re-published.

\section{Manuscript Withdrawal Policy}

Authors must abide by these policies after submission of their manuscript.

- Authors must provide their consent to publish their manuscript prior to peerreview process.

- Withdrawing an already confirmed manuscript after it has been accepted requires consent from all the authors with valid reason for withdrawing of their manuscript.

\section{Availability of Materials and Data}

Supporting data must be made available to Editorial Board Members and referees at the time of submission for the purposes of evaluating the manuscript. Referees may be asked to comment on the terms of access to materials, methods and/or data sets; GE reserves the right to refuse publication in cases where authors do not provide adequate assurances that they can comply with the publication's requirements for sharing materials.

\section{Disclaimer}

The author(s) of each article appearing in this Journal is/are solely responsible for the content thereof; neither the Geo Eye nor its editors or publisher or anyone else involved in creating, producing or distribution assumes any liability or responsibility for the accuracy, completeness, or usefulness of any information provided in the Journal, nor shall they be liable for any direct, indirect, incidental, special, consequential or punitive damages arising out of the use of Geo Eye.

\section{Plagiarism}

Plagiarism is unacknowledged copying or an attempt to misattribute original authorship, whether of ideas or text. As defined by the ORI (Office of Research Integrity), plagiarism can include, "theft or misappropriation of intellectual property and the substantial unattributed textual copying of another's work". Plagiarism can be said to have clearly occurred when large chunks of text have been cut-and-pasted without appropriate and unambiguous attribution. Such manuscripts would not be considered for publication in GA. When re-using text, whether from the author's own publication or that of others, appropriate attribution and citation is necessary to avoid creating a misleading perception of unique contribution for the reader. 
GE Editors and Editorial Board members assess all such

cases on their individual merits. When plagiarism becomes evident post-publication, we may correct or retract the original publication depending on the degree of plagiarism, context within the published article and its impact on the overall integrity of the published study.

For every Journal, the peer review process is at the heart of the success of publishing. As part of our commitment to the protection and enhancement of the peer review process, The Geo Eye would like to ensure that all published articles are within the accepted level of plagiarism. The Journal uses the very well and widely accepted Plagiarism Detection tool, iThenticate. All submitted Manuscripts are first subjected to Plagiarism check and then the Publishing cycle begins.

In case, plagiarism is detected during review/editorial process, such manuscript(s) will be rejected immediately and we can also consider other steps depending upon the seriousness of the case. If the plagiarism is proven after publication, such manuscript(s) will be removed from our website and appropriate announcement will be placed in this regards. Further, Geo Eye can bring such instances in notice of author's funding agencies, author's institutes (where they work) and to the original authors whose work has been plagiarized. Please report the plagiarism to info@sciresol.com

\section{Competing Interest}

In the interests of transparency and to help readers to form their own judgments of potential bias, authors must declare any competing financial and/or non-financial interests in relation to the work described.

Competing interests are defined as financial and non-financial interests that could directly undermine, or be perceived to undermine, the objectivity, integrity and value of a publication, through a potential influence on the judgements and actions of authors with regard to objective data presentation, analysis and interpretation.

\section{a. Financial Competing Interest}

i. Funding: Research support (including salaries, equipment, supplies, and other expenses) by organizations that may gain or lose financially through this publication. A specific role for the funder in the conceptualization, design, data collection, analysis, decision to publish, or preparation of the manuscript, should be disclosed. 


\section{GE Journal Policies}

ii. Employment: Recent (while engaged in the research project), present or anticipated employment by any organization that may gain or lose financially through this publication.

iii. Personal financial interests: Stocks or shares in companies that may gain or lose financially through publication; consultation fees or other forms of remuneration (including reimbursements for attending symposia) from organizations that may gain or lose financially; patents or patent applications (awarded or pending) filed by the authors or their institutions whose value may be affected by publication. For patents and patent applications, disclosure of the following information is requested: patent applicant (whether author or institution), name of inventor(s), application number, status of application, specific aspect of manuscript covered in patent application.

\section{b. Non-financial Competing Interest}

Non-financial competing interests can take different forms, including personal or professional relations with organizations and individuals. We would encourage authors and referees to declare any unpaid roles or relationships that might have a bearing on the publication process. Examples of non-financial competing interests include (but are not limited to):

- Unpaid membership in a government or non-governmental organization

- Unpaid membership in an advocacy or lobbying organization

- Unpaid advisory position in a commercial organization

- Writing or consulting for an educational company

- Acting as an expert witness

The corresponding author is responsible for submitting a competing interest's statement on behalf of all authors of the paper. We do not require authors to state the monetary value of their financial interests.

GE request the peer-reviewers to exclude themselves in cases where there is a significant conflict of interest, financial or otherwise. However, just as financial interests need not invalidate the conclusions of an article, nor do they automatically disqualify an individual from evaluating it. We ask peer-reviewers to inform the editors of any related interests, including financial interests as defined above that might be perceived as relevant.

Editorial Board Members are required to declare any interests that might influence, or be perceived to influence, their editorial activities. In particular, they should 
exclude themselves from handling manuscripts in cases

where there is a conflict of interest, financial or otherwise. This may include - but is not limited to - having previously published with one or more of the authors, and sharing the same institution as one or more of the authors.

Editorial Board Members are welcome to submit papers to the journal. These submissions are not given any priority over other manuscripts, and Editorial Board Member status has no bearing on editorial consideration. When an Editorial Board Member is an author on a submission, they will have no involvement in the editorial review of the manuscript, no access to confidential information on the editorial process, and no input into the decision to publish the article.

\section{Article Retraction}

Editor's decision is the decisive factor for all scholarly publications. However, this decision is based on Editorial Policies - which includes plagiarism, copyright etc. It becomes important to retain the Published articles as it is - without any alterations. However, owing to certain circumstances, a published article may at times be retracted, removed or may require a change.

Retraction could be due to:

- Plagiarism

- Bogus Authorship

- Fraudulent Data

- Correction in Publication

The article PDF will remain available with the watermark "Retracted" available on it. Only in case of legal/controversial/incorrect PDF, they will be replaced or removed completely from the website with a note.

\section{Contact}

Dr. Ashok D Hanjagi

Editor in Chief

Professor, Department of Geography

Bangalore University, Bangalore

Email: ashokhanjagi@bub.ernet.in 